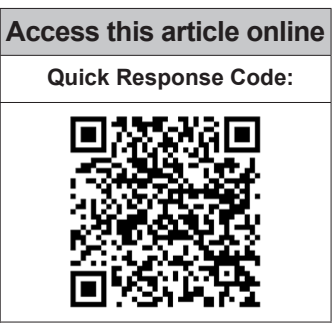

Website:

www.jponline.org

DOI:

10.4103/JLP.JLP_136_19
Department of Microbiology, All India Institute of Medical Sciences, Bhubaneswar, Odisha, India

Address for correspondence: Dr. Baijayantimala Mishra,

Department of Microbiology, All India Institute of Medical Sciences, Bhubaneswar - 751019 , Odisha, India E-mail:bm_mishra@ hotmail.com

Submission: 20-08-2019 Accepted: 27-11-2019

\title{
Detection of carbapenemase-producing Pseudomonas aeruginosa by phenotypic and genotypic methods in a tertiary care hospital of East India
}

\author{
Nishu Verma, Ashok Kumar Prahraj, Baijayantimala Mishra, Bijayini Behera, \\ Kavita Gupta
}

\section{Abstract:}

BACKGROUND: Carbapenemase-producing Pseudomonas aeruginosa is a serious threat in hospital infection due to its multidrug resistance.

AIM: The aim of the study was to determine the frequency of carbapenem resistance in clinical isolates of Pseudomonas aeruginosa and detect the presence of carbapenemase enzymes in carbapenemresistant $P$. aeruginosa (CRPA) isolates by phenotypic and genotypic methods.

MATERIAL AND METHODS: Double-disk synergy test [DDST] and combined disk synergy test [CDST]) was performed in CRPA isolates and the prevalence of blaKPC, bla NDM-1, blalMP, bla VIM, blasIM, blaSPM, bla GIM, and bla OXA-48 was determined.

RESULTS: Of 559 isolates included in the study, a total of 102 isolates were resistant to carbapenem that accounted for overall $18.24 \%$ (102/559) prevalence. Of these 102 isolates, $89(87.25 \%)$ isolates were positive by DDST and $95(93.17 \%)$ isolates were positive by CDST. Of 102 CRPA isolates, bla VIM was detected in 30 isolates (30/102, 29.1\%), followed by bla NDM-1 in $29(29 / 102,28.4 \%)$ isolates and blasIM and bla GIM in 6 isolates each (6/102, 5.8\%). A combination of two carbapenemase genes was detected in 12 isolates, with six (6/102, 5.88\%) CRPA isolates harboring with both blaVIM and ${ }^{\text {bla NDM-1 }}$ genes. Four isolates were found to harbor a combination of three carbapenem-resistant genes.

CONCLUSION: A high rate of carbapenemase production was observed in $P$. aeruginosa. Coproducers of multiple carbapenemases are also a cause of concern. An in-depth understanding of molecular mechanisms of resistance will be helpful in optimizing patient management and hospital infection control.

Key words:

Carbapenemases, genotypic methods, phenotypic methods, Pseudomonas aeruginosa

\section{Introduction}

Cart arbapenem-resistant Pseudomonas aeruginosa (CRPA) is listed as an organism posing a serious threat by the Centers for Disease Control and Prevention..$^{[1]}$ In India, up to $40 \% P$. aeruginosa isolates are shown to be carbapenem resistant. ${ }^{[2]}$ Carbapenem resistance in $P$. aeruginosa has shown to

This is an open access journal, and articles are distributed under the terms of the Creative Commons Attribution-NonCommercial-ShareAlike 4.0 License, which allows others to remix, tweak, and build upon the work non-commercially, as long as appropriate credit is given and the new creations are licensed under the identical terms.

For reprints contact: reprints@medknow.com be multifactorial, including production of carbapenemase, overexpression/ overproduction of efflux pump, and porin loss. Carbapenemases in P. aeruginosa belong to three molecular classes, for example, class A ( $\left.{ }^{\text {bla }} \mathrm{KPC}\right)$, class B ( ${ }^{\text {bla }} \mathrm{IMP}$, bla VIM, bla NDM, blaSIM, bla GIM, and blaSPM), and class D (bla OXA-48) genes. The molecular mechanism of carbapenem resistance in $P$. aeruginosa has been studied in very few studies restricted to North and South India. ${ }^{[2,3]}$

How to cite this article: Verma N, Prahraj AK, Mishra B, Behera B, Gupta K. Detection of carbapenemase-producing Pseudomonas aeruginosa by phenotypic and genotypic methods in a tertiary care hospital of East India. J Lab Physicians 2019;11:287-91. 
The aim of the present study was to determine the production of carbapenemase enzymes in CRPA isolates by phenotypic methods (double-disk synergy test [DDST] and combined disk synergy test [CDST]) and to determine the prevalence of bla KPC, blaNDM-1, bla IMP, bla VIM, blaSIM, blaSPM, bla GIM, and bla OXA-48. The susceptibility profile of $P$. aeruginosa to other antipseudomonal antibiotics was also investigated.

\section{Materials and Methods}

This study was conducted at the microbiology department of a tertiary care teaching hospital from January 2017 to May 2018. All consecutive nonduplicate isolates of $P$. aeruginosa from different clinical samples (blood, respiratory sample, pus, urine, sterile body fluids, and other samples) resistant to meropenem and/or imipenem by disk diffusion method were included in the study. The study was conducted after ethical clearance from the Institutional Ethics Committee (ref no-IEC/ AIIMS BBSR/PG Thesis/2017-18/3).

The initial isolation and identification of $P$. aeruginosa were carried out by standard bacteriological procedures. The following antimicrobials were tested by disk diffusion: ceftazidime $(30 \mu \mathrm{g})$, cefepime $(30 \mu \mathrm{g})$, piperacillintazobactam $(100 \mu \mathrm{g} / 10 \mu \mathrm{g})$, ticarcillin-clavulanate $(75 \mu \mathrm{g} / 10 \mu \mathrm{g})$, imipenem $(10 \mu \mathrm{g})$, meropenem $(10 \mu \mathrm{g})$, doripenem $(10 \mu \mathrm{g})$, amikacin $(30 \mu \mathrm{g})$, gentamicin $(10 \mu \mathrm{g})$, netilmicin $(30 \mu \mathrm{g})$, tobramycin $(10 \mu \mathrm{g})$, ciprofloxacin $(5 \mu \mathrm{g})$, and levofloxacin $(5 \mu \mathrm{g})$ (HiMedia Laboratories Pvt. Ltd., Mumbai, Maharashtra, India). The antimicrobial susceptibility was interpreted as per the Clinical and Laboratory Standards Institute 2017 guidelines using $P$. aeruginosa ATCC strain 27853 as quality control strain. ${ }^{[4]}$ In addition, imipenem and meropenem minimum inhibitory concentration was determined by agar dilution and E-test (HiMedia Laboratories Pvt. Ltd., Mumbai, Maharashtra, India).

Isolates with imipenem/meropenem/doripenem MIC $\geq 8 \mu \mathrm{g} / \mathrm{ml}$ by agar dilution and E-test were screened for carbapenemase production by DDST and CDST by the following methods.

- Double-disk synergy test: ${ }^{[5]}$ As described by Lee et al., this test was carried out using ethylenediaminetetraacetic acid (EDTA) disk $(10 \mu \mathrm{l}$ of $0.5 \mathrm{M}$ EDTA) and the imipenem disk $(10 \mu \mathrm{g})$ using the standard method. ${ }^{[5]}$ The production of large synergistic inhibitory zones was interpreted as test positive for metallo-beta-lactamase (MBL)

- Combined disk synergy test: ${ }^{[6]}$ As described by Yong et al., this test was carried out using two imipenem commercial disks with and without EDTA $(10 \mu \mathrm{l}$ of $0.5 \mathrm{M}$ EDTA). ${ }^{[6]}$ The zone of inhibition diameter with imipenem + EDTA disk $\geq 7 \mathrm{~mm}$ than the imipenem disk alone was considered positive for MBL production.

Molecular detection of carbapenemase gene Multiplex polymerase chain reaction (PCR) for the presence of bla $\mathrm{KPC}$, bla NDM-1, bla IMP, bla VIM, blaSIM, blaSPM, ${ }^{\text {bla }} \mathrm{GIM}$, and bla OXA-48 was carried out according to the method published by Ellington et al.$^{[7]}$ and Kumarasamy et al. ${ }^{[8]}$ Briefly, DNA extraction from $P$. aeruginosa isolates was carried out using QIAamp DNA Mini kit spin-column method (QIAGEN, Hilden, Germany) as per manufacturer's instruction. Two sets of multiplex PCR were done using KAPA2G Fast Multiplex PCR Kit (2X) as per manufacturer's instructions (Kapa Biosystems, Cape Town, South Africa). The first set of multiplex PCR detected blaMP, VIM, GIM, SPM, and SIM and the second set of multiplex PCR detected bla NDM-1, OXA-48, and KPC. The PCR reactions consisted of a final reaction volume of $25 \mu \mathrm{l}$ containing $5 \mu \mathrm{l}$ of template DNA, $12.5 \mu \mathrm{l}$ of 2X KAPA2G Fast Multiplex Master Mix, and $0.5 \mu \mathrm{l}$ of each oligonucleotide primer. Amplification was done with initial denaturation step at $95^{\circ} \mathrm{C}$ for $3 \mathrm{~min}$, followed by denaturation at $95^{\circ} \mathrm{C}$ for $15 \mathrm{~s}$, annealing at $60^{\circ} \mathrm{C}$ for $30 \mathrm{~s}$, extension at $72^{\circ} \mathrm{C}$ for $30 \mathrm{~s}$, and a final extension step at $72^{\circ} \mathrm{C}$ for $7 \mathrm{~min}$. The product was visualized by $1.5 \%$ agarose gel electrophoresis, stained with ethidium bromide, and was viewed under automated gel documentation system (Syngene G: Box, Syngene, Cambridge, U.K.) using ultraviolet illumination. The primer sequences and the product sizes $^{[7,8]}$ are given in Table 1.

\section{Statistical analysis}

The sensitivity and specificity of CDST and DDST were calculated by forming $2 \times 2$ contingency tables and genotypic tests being considered as a gold standard.

Table 1: Primers used for multiplex polymerase chain reaction for various carbapenemase genes with their product size

\begin{tabular}{llc}
\hline Primer & \multicolumn{1}{c}{ Primer sequence (5'-3') } & Product size (bp) \\
\hline IMP family-F GGA ATA GAG TGG CTT AAY TCT C & 188 \\
IMP family-RCCA AAC YAC TAS GTT ATC T & \\
$\begin{array}{l}\text { VIM family-F GAT GGT GTT TGG TCG CAT A } \\
\text { VIM family-RCGA ATG CGC AGC ACC AG }\end{array}$ & 390 \\
GIM-1-F & TCG ACA CAC CTT GGT CTG AA & 477 \\
GIM-1-R & AAC TTC CAA CTT TGC CAT GC & \\
SPM-1A-F & AAA ATC TGG GTA CGC AAA CG & 271 \\
SPM-1A-R & ACA TTA TCC GCT GGA ACA GG & \\
SIM-1-F & TAC AAG GGA TTC GGC ATC G & 571 \\
SIM-1-R & TAA TGG CCT GTT CCC ATG TG & \\
NDM-1 F & ACC GCC TGG ACC GAT GAC CA & 264 \\
NDM-1 R & GCC AAA GTT GGG CGC GGT TG & \\
OXA-48-F & TTGGTGGCATCGATTATCGG & 744 \\
OXA-48-R & GAGCACTTCTTTTGTGATGGC & \\
KPC-F & ATGTCACTGTATCGCCGTCT & 893 \\
KPC-R & TTTTCAGAGCCTTACTGCCC & \\
\hline
\end{tabular}




\section{Results}

A total of 559 consecutive nonduplicate isolates of $P$. aeruginosa were recovered from various clinical samples received in the department of microbiology during the study period (January 2017-May 2018). Of 559 isolates of P. aeruginosa, a total of 102 isolates $(102 / 559,18.2 \%)$ were resistant to carbapenem (MIC $\geq 8 \mu \mathrm{g} / \mathrm{ml}$ ). Of these 102 isolates, $19(19 / 102,18.6 \%)$ were from various intensive care units, $24(24 / 102,23.5 \%)$ were from various outpatient departments, and rest $59(59 / 102,57.8 \%)$ were from various admitted patients. CRPA was isolated from pus $(38 / 102,37.2 \%)$, followed by urine $(31 / 102,30.3 \%)$, respiratory samples $(25 / 102,24.5 \%)$, blood $(3 / 102$, $2.9 \%)$, and other samples $(5 / 102,4.9 \%)$, respectively. Among the various antipseudomonal antibiotics tested in CRPA isolates, the maximum susceptibility was observed for piperacillin-tazobactam $(17 / 102$, $16.6 \%)$, followed by ceftazidime $(12 / 102,11.7 \%)$ and aminoglycosides (8/102, 7.8\%). Ticarcillin-clavulanate with a percentage susceptibility of $2.9 \%(3 / 102)$ was found to be least susceptible. All CRPA isolates were found to be resistant to ciprofloxacin and levofloxacin. Of 102 CRPA isolates, CDST and DDST were positive in $95(93.1 \%)$ and $89(87.2 \%)$, respectively, as shown in Table 2. Figures 1 and 2 depict the positive CDST and DDST, respectively.

Of 102 CRPA isolates, bla VIM was detected in 30 isolates $(30 / 102,29.1 \%)$, followed by bla NDM-1 in $29(29 / 102,28.4 \%)$ isolates and blasIM and bla GIM in 6 isolates each $(6 / 102,5.8 \%)$. A combination of two carbapenemase genes were detected in 12 isolates, with six $(6 / 102,5.88 \%)$ CRPA isolates harboring with both bla VIM and blaNDM-1 genes. Four isolates were found to harbor a combination of three carbapenem-resistant genes [Figure 3]. No CRPA isolates were found to possess blaIMP, bla KPC, and bla OXA-48. The distribution of various carbapenemase genes in 102 CRPA isolates is provided in Table 2. Five isolates of CRPA that were positive for NDM-1 were sent for sequencing by Sanger's method to Eurofins Genomics India Pvt. Ltd., Bengaluru, Karnataka, India, and interpreted using BLAST software (NIH, USA), of which three were NDM-1 and two were found to be NDM-5. The sensitivity of DDST and CDST was found calculated taking the presence of any MBL gene as a reference test and was found to be $81.35 \%$ and $92.18 \%$, respectively, as shown in Table 3.

\section{Discussion}

Carbapenem resistance was noted in $18.2 \%$ P. aeruginosa isolates in our study. In the Indian Council of Medical Research scoping document, the prevalence of CRPA isolates across Indian hospitals is shown to be $42 \%-47 \%$ in the years 2014 and 2015. ${ }^{[9]}$ In a recent single-center study from North India, the prevalence of CRPA is shown to be $72.5 \%$ in admitted patients. ${ }^{[10]}$ The relatively lower prevalence of CRPA in our study could be due to

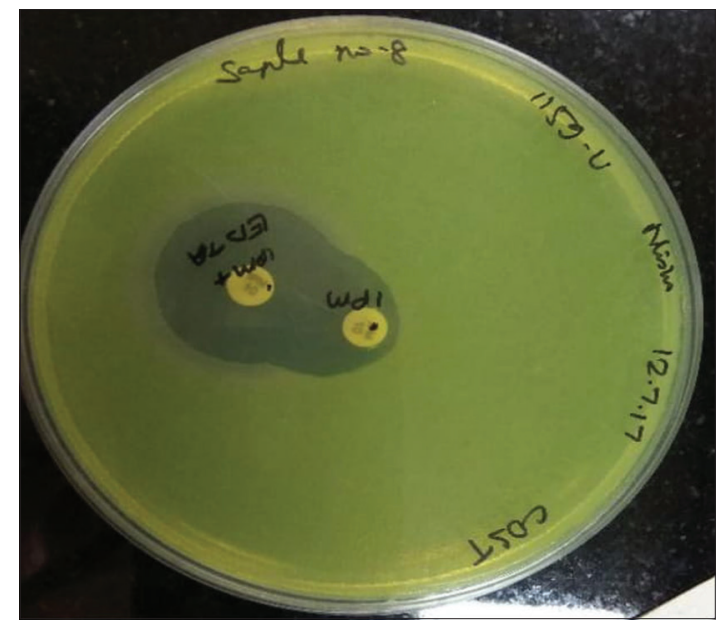

Figure 1: Isolate showing positive carbapenemase-producing Pseudomonas aeruginosa test with $\geq 7 \mathrm{~mm}$ increase in the zone of inhibition of imipenem + ethylenediaminetetraacetic acid as compared to imipenem alone

Table 2: Distribution of various carbapenem-resistant genes in carbapenem-resistant Pseudomonas aeruginosa isolates $(n=102)$

\begin{tabular}{|c|c|c|c|c|c|}
\hline Type of carbapenemase gene identified & Number of isolates $(\%),(n=102)$ & CDST+ & CDST- & DDST+ & DDST- \\
\hline blanDM-1 & $19(18.62)$ & 19 & 0 & 15 & 4 \\
\hline blayIM & $17(16.62)$ & 16 & 1 & 14 & 3 \\
\hline blasIM & $6(5.88)$ & 5 & 1 & 6 & 0 \\
\hline blaGIM & $6(5.88)$ & 4 & 2 & 5 & 1 \\
\hline bla VIM+bla NDM-1 & $6(5.88)$ & 6 & 0 & 5 & 1 \\
\hline bla VIM+ $+{ }^{\text {bla }}$ GIM & $3(2.94)$ & 3 & 0 & 3 & 0 \\
\hline 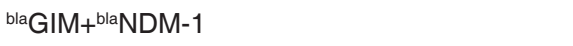 & $2(1.96)$ & 2 & 0 & 2 & 0 \\
\hline bla GIM+bla SPM & $1(0.98)$ & 1 & 0 & 0 & 1 \\
\hline 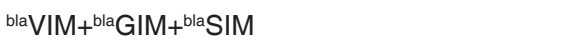 & $1(0.98)$ & 0 & 1 & 1 & 0 \\
\hline bla VIM $+{ }^{\text {bla }}$ SPM $+{ }^{\text {bla }}$ NDM-1 & $1(0.98)$ & 1 & 0 & 0 & 1 \\
\hline bla VIM+bla GIM+bla NDM-1 & $1(0.98)$ & 1 & 0 & 1 & 0 \\
\hline bla VIM+bla GIM+bla SPM & $1(0.98)$ & 1 & 0 & 1 & 0 \\
\hline Total & 64 & 59 & 5 & 53 & 11 \\
\hline
\end{tabular}

CDST=Combined disk synergy test 


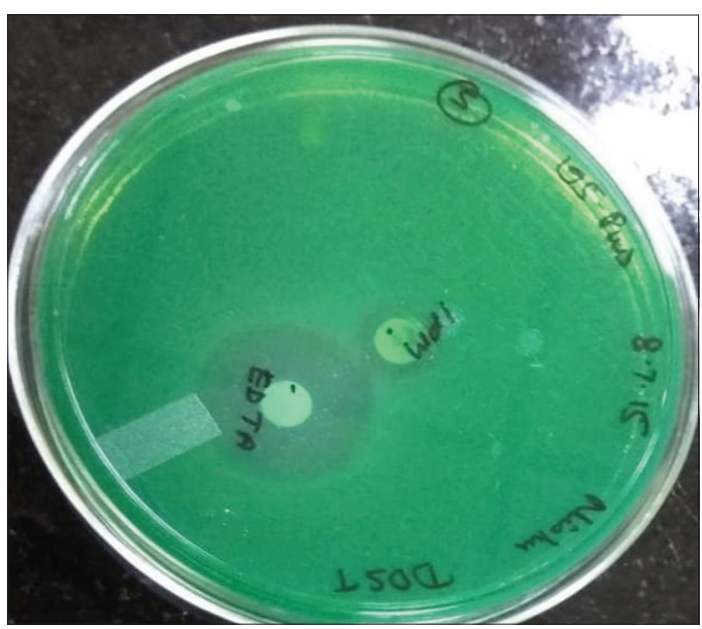

Figure 2: Isolate showing positive double-disk synergy test with enhanced zone of inhibition around ethylenediaminetetraacetic acid as compared to imipenem

Table 3: Comparison of different phenotypic test in metallo-beta-lactamase-producing Pseudomonas aeruginosa $(n=102)$

\begin{tabular}{lccc}
\hline Type of test & Positive (\%) & Negative (\%) & Sensitivity (\%) \\
\hline DDST test & $89(87.25)$ & $13(12.75)$ & 81.35 \\
CDST test & $95(93.17)$ & $7(6.83)$ & 92.18
\end{tabular}

DDST=Double-disk synergy test, CDST=Combined disk synergy test

the higher proportion of isolates being included from outpatient settings.

In our study, the sensitivity of DDST and CDST was found to be $81.35 \%$ and $92.18 \%$, respectively (taking the presence of any MBL gene as a reference test). The sensitivity of CDST for MBL detection was shown to be $86.7 \%$ and specificity of $51.1 \%$ in a previous study by Peter et al. in 2014. ${ }^{[11]}$ The higher sensitivity of CDST compared to DDST, as observed in our study, is also described previously. ${ }^{[12]}$ In the present study, the prevalence of MBL was $15.74 \%$ by phenotypic method (CDST), whereas the prevalence by genotypic method for MBL was found as $10.19 \%$. The concordance rate between CDST and genotypic method of MBL detection in our study was found to be $92.18 \%$. The overall rate of concordance between phenotypic and genotypic tests for the detection of MBL was $98 \%$ in a study by Kazi et al. ${ }^{[13]}$

In our study, $62.74 \%$ CRPA isolates had at least one carbapenemase gene. bla VIM was the most prevalent gene in CRPA (30/64, 46.87\%), followed by bla NDM-1 (29/64, $45.31 \%)$. bla VIM and bla NDM are shown to the most prevalent carbapenemase gene in the majority of Indian studies. ${ }^{[2,3,12,14]}$ In the study by Ellapan et al. in 2018 , bla VIM and bla NDM were present in $23.1 \%$ and $17.3 \%$ of 156 CRPA isolates. ${ }^{[15]}$ In another study by Mohanam and Menon in 2017, bla VIM and bla NDM were present in $32 \%$ and $27 \%$ of 213 CRPA isolates. ${ }^{[16]}$ In a multicentric study from India, bla VIM and bla NDM were

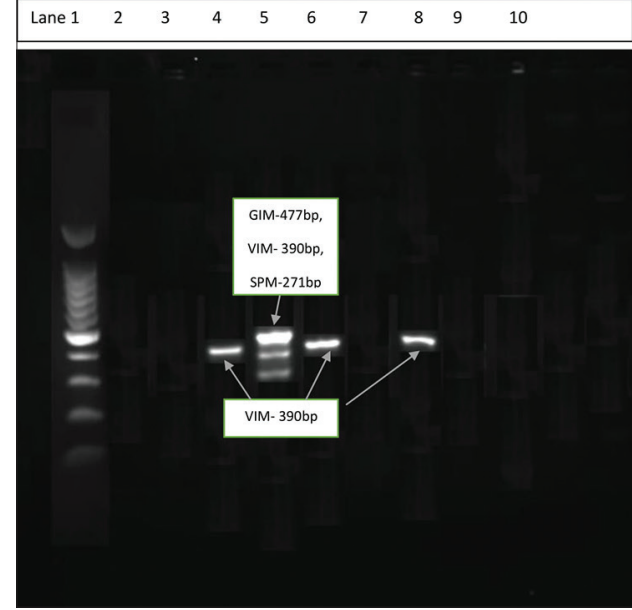

Figure 3: Multiplex polymerase chain reaction - Lane 1 showing $100 \mathrm{bp}$ ladder; Lanes 4, 6, and 8 showing blaVIM-390 bp; and Lane 5 showing bla GIM + blaVIM + blasPM

the most frequent carbapenemases in CRPA isolates with regional differences in the carbapenemase profile across the study sites. ${ }^{[2]}$ The incidence of CRPA has increased worldwide, and they pose a potential risk for therapeutic failure with the empirical treatments currently in place. In this study, no bla KPC, bla IMP, and

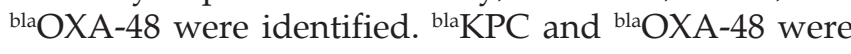
also absent in 156 CRPA isolates analyzed by Ellapan et al. ${ }^{[15]}$ In another multicentric study by Khurana et al., ${ }^{b l a} \mathrm{KPC}$ and bla OXA-1 were present in $43 \%$ and $56 \%$ of CRPA isolates from North India but were absent in CRPA isolates collected from South India. ${ }^{[17]}$ In the present study, 15 CRPA isolates had coexistence of two or three carbapenem-resistant genes. ${ }^{\text {bla }}$ VIM $+{ }^{\text {bla }} \mathrm{NDM}-1$ was the most common combination $(6 / 102,5.88 \%)$, followed by bla VIM + bla GIM (3/102, 2.94\%). The coproduction of bla VIM + bla NDM-1 had previously been described in $7.1 \%$ CRPA isolates by Ellapan et al. ${ }^{[15]}$ The coexistence of bla VIM + ${ }^{\text {bla }} \mathrm{NDM}-1$ and bla KPC-2 and bla NDM-1 in CRPA isolates from India is also described previously by Paul et al. ${ }^{[18,19]}$

No carbapenem-resistant gene was detected in 38 (38/102, $37.25 \%$ ) CRPA isolates in our study. Other carbapenem mechanisms such as efflux pump and porin loss could have contributed for carbapenemase resistance, which were not studied in the present study. Loss of OprD porin and overexpression of mexA gene is also found in studies from India either as a standalone mechanism or in association with the presence of bla VIM. ${ }^{[15]}$

In our study, fluoroquinolones did not have any activity against CRPA isolates. Polymyxin B and colistin were found to be susceptible in all isolates of $P$. aeruginosa. There are recent studies of colistin resistance in $<5 \%$ $P$. aeruginosa isolates from India, which warrant judicious use of this last-resort antibiotic. ${ }^{[14]}$ Among 
beta-lactam and beta-lactamase inhibitor combination, the maximum susceptibility was observed for piperacillin-tazobactam $(16.67 \%)$, followed by ceftazidime with $11.76 \%$ and $2.94 \%$ for ticarcillin-clavulanate which was found to be least susceptible. Carbapenem-resistant and cephalosporin-susceptible $P$. aeruginosa is a rare phenotype. Previous carbapenem therapy and decreased OprD expression and efflux system overexpression are cited as possible mechanisms of this phenotype. The clinical utility in this group is, however, debatable. ${ }^{[20]}$

\section{Conclusion}

A high rate of carbapenemase production was observed in P. aeruginosa. Coproducers of multiple carbapenemases are also a cause of concern. An in-depth understanding of molecular mechanisms of resistance will be helpful in optimizing patient management and hospital infection control.

\section{Financial support and sponsorship \\ Nil.}

\section{Conflicts of interest}

There are no conflicts of interest.

\section{References}

1. Centers for Disease Control and Prevention, Office of Infectious Diseases. Antibiotic Resistance Threats in the United States, 2013. Atlanta, GA: Centers for Disease Control and Prevention, Office of Infectious Diseases; 2013. Available from: http:/ / www.cdc.gov/ drugresistance/threat-report-2013. [Last accessed on 2019 Jul 24].

2. Pragasam AK, Veeraraghavan B, Anandan S, Narasiman V, Sistla S, Kapil A, et al. Dominance of international high-risk clones in carbapenemase-producing Pseudomonas aeruginosa: Multicentric molecular epidemiology report from India. Indian J Med Microbiol 2018;36:344-51.

3. Pragasam AK, Vijayakumar S, Bakthavatchalam YD, Kapil A, Das BK, Ray P, et al. Molecular characterisation of antimicrobial resistance in Pseudomonas aeruginosa and Acinetobacter baumannii during 2014 and 2015 collected across India. Indian J Med Microbiol 2016;34:433-41.

4. Clinical and Laboratory Standards Institute. Performance Standards for Antimicrobial Susceptibility Testing. $27^{\text {th }}$ ed. CLSI Document M100. Wayne, PA: Clinical and Laboratory Standards Institute; 2017.

5. Lee K, Lim YS, Yong D, Yum JH, Chong Y. Evaluation of the hodge test and the imipenem-EDTA double-disk synergy test for differentiating metallo-beta-lactamase-producing isolates of Pseudomonas spp. and Acinetobacter spp. J Clin Microbiol 2003;41:4623-9.

6. Yong D, Lee K, Yum JH, Shin HB, Rossolini GM,
Chong Y. Imipenem-EDTA disk method for differentiation of metallo-beta-lactamase-producing clinical isolates of Pseudomonas spp. and Acinetobacter spp. J Clin Microbiol 2002;40:3798-801.

7. Ellington MJ, Kistler J, Livermore DM, Woodford N. Multiplex PCR for rapid detection of genes encoding acquired metallo-beta-lactamases. J Antimicrob Chemother 2007;59:321-2.

8. Kumarasamy KK, Toleman MA, Walsh TR, Bagaria J, Butt F, Balakrishnan R, et al. Emergence of a new antibiotic resistance mechanism in India, Pakistan, and the UK: A molecular, biological, and epidemiological study. Lancet Infect Dis 2010;10:597-602.

9. Gandra S, Joshi J, Trett A, Lamkang AS, Laxminarayan R. Scoping report on antimicrobial resistance in India. Washington, DC: Center for Disease Dynamics, Economics and Policy; 2017.

10. Bajpai V, Govindaswamy A, Khurana S, Batra P, Aravinda A, Katoch $\mathrm{O}$, et al. Phenotypic genotypic profile of antimicrobial resistance in Pseudomonas species in hospitalized patients. Indian J Med Res 2019;149:216-21.

11. Peter S, Lacher A, Marschal M, Hölzl F, Buhl M, Autenrieth I, et al. Evaluation of phenotypic detection methods for metallo- $\beta$-lactamases (MBLs) in clinical isolates of Pseudomonas aeruginosa. Eur J Clin Microbiol Infect Dis 2014;33:1133-41.

12. Behera B, Mathur P, Das A, Kapil A, Sharma V. An evaluation of four different phenotypic techniques for detection of metallo-beta-lactamase producing Pseudomonas aeruginosa. Indian J Med Microbiol 2008;26:233-7.

13. Kazi M, Nikam C, Shetty A, Rodrigues C. Dual-tubed multiplex-PCR for molecular characterization of carbapenemases isolated among Acinetobacter spp. and Pseudomonas spp. J Appl Microbiol 2015;118:1096-102.

14. Veeraraghavan B, Pragasam AK, Bakthavatchalam YD, Anandan S, RamasubramanianV, Swaminathan S, et al. Newer B-Lactam/ B-Lactamase inhibitor for multidrug-resistant gram-negative infections: Challenges, implications and surveillance strategy for India. Indian J Med Microbiol 2018;36:334-43.

15. Ellappan K, Belgode Narasimha H, Kumar S. Coexistence of multidrug resistance mechanisms and virulence genes in carbapenem-resistant Pseudomonas aeruginosa strains from a tertiary care hospital in South India. J Glob Antimicrob Resist 2018;12:37-43.

16. Mohanam L, Menon T. Coexistence of metallo-beta-lactamaseencoding genes in Pseudomonas aeruginosa Indian J Med Res 2017;146:S46-52.

17. Khurana S, Mathur P, Kapil A, Valsan C, Behera B. Molecular epidemiology of beta-lactamase producing nosocomial Gram-negative pathogens from North and South Indian hospitals. J Med Microbiol 2017;66:999-1004.

18. Paul D, Dhar Chanda D, Maurya AP, Mishra S, Chakravarty A, Sharma GD, et al. Co-Carriage of blaKPC-2 and blaNDM-1 in clinical isolates of Pseudomonas aeruginosa Associated with hospital infections from India. PLoS One 2015;10:e0145823.

19. Paul D, Dhar D, Maurya AP, Mishra S, Sharma GD, Chakravarty A, et al. Occurrence of co-existing bla VIM-2 and blaNDM-1 in clinical isolates of Pseudomonas aeruginosa from India. Ann Clin Microbiol Antimicrob 2016;15:31.

20. Li S, Jia X, Li C, Zou H, Liu H, Guo Y, et al. Carbapenem-resistant and cephalosporin-susceptible Pseudomonas aeruginosa: A notable phenotype in patients with bacteremia. Infect Drug Resist 2018;11:1225-35. 